\title{
Grons

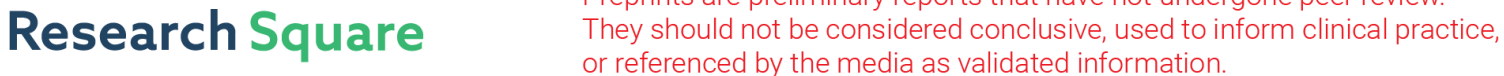

\section{Autophagy Activation Induces Resistance to FLT3 Inhibitors in Acute Myeloid Leukemia with FLT3-ITD Mutation}

\section{Dan Xu}

Southern Medical University Nanfang Hospital

Zhao Yin

Southern Medical University Nanfang Hospital

\section{Ying Yang}

Southern Medical University Nanfang Hospital

\section{Yishan Chen}

Southern Medical University Nanfang Hospital

\section{Changfen Huang}

Southern Medical University Nanfang Hospital

\section{Qiang Wang}

Southern Medical University Nanfang Hospital

\section{Ling Jiang}

Southern Medical University Nanfang Hospital

\section{Xuejie Jiang}

Southern Medical University Nanfang Hospital

\section{Changxin Yin}

Southern Medical University Nanfang Hospital

Qifa Liu

Southern Medical University Nanfang Hospital

Guopan Yu ( $\nabla$ yugpp@163.com )

Southern Medical University Nanfang Hospital https://orcid.org/0000-0002-5732-0318

\section{Research}

Keywords: autophagy, FLT3 inhibitor, resistance, acquired mutation, bone marrow micro-environment, FLT3-ITD, acute myeloid leukemia

Posted Date: July 9th, 2021

DOI: https://doi.org/10.21203/rs.3.rs-676343/v1 
License: (c) (i) This work is licensed under a Creative Commons Attribution 4.0 International License. Read Full License 


\section{Abstract}

Background: Autophagy plays a critical role in drug resistance in acute myeloid leukemia (AML), including the subtype with FLT3-ITD mutation. Yet how autophagy is activated and mediates resistance to FLT3 inhibitors in FLT3-ITD-positive AML remains unsure.

Methods: We detected the alteration of autophagy in FLT3-ITD-positive leukemic cells after versus before acquired resistance to FLT3 inhibitors; tested the stimulative effect of acquired D835Y mutation and bone marrow micro-environment (BME) on autophagy; explored the mechanism of autophagy mediating FLT3 inhibitor resistance.

Results: Sorafenib-resistant cells markedly overexpressed autophagy in comparison with sorafenibsensitive cells or the cells before sorafenib treatment. Both acquired D835Y mutation and BME activated cytoprotective autophagy to induce FLT3 inhibitor resistance. Autophagy activation decreased the suppression efficacy of FLT3 inhibitors on FLT3 downstream signaling and then weakened their antileukemia effect. Inhibition of autophagy with CQ significantly enhanced the suppressive effect of FLT3 inhibitor on FLT3 downstream signaling, in the end overcame FLT3 inhibitor resistance.

Conclusions: Autophagy might be stimulated by acquired mutation or BME, and bypass activate FLT3 downstream signaling to mediate FLT3 inhibitor resistance in FLT3-ITD-positive AML. Targeting autophagy could be a promising strategy to overcome resistance.

\section{Background}

Fms-like tyrosine kinase 3-internal tandem duplication (FLT3-ITD) mutation is a common molecular event with an approximate incidence of $25 \%$ in acute myeloid leukaemia (AML) ${ }^{[1]}$. High allelic ratio $(\geq 0.5)$ of FLT3-ITD is associated with a very poor prognosis in both adults and children, and are rarely cured by chemotherapy alone ${ }^{[2-3]}$. Incorporation of FLT3 inhibitors to target FLT3 pathway with chemotherapy or haematopoietic stem cell transplantation has significantly improved the prognosis of FLT3-ITD mutant $A M L$ recently years ${ }^{[4-6]}$, but leukaemia relapse remains high ${ }^{[2-3]}$. Resistance to FLT3 inhibitors plays an important role in leukemia relapse ${ }^{[2,7-8]}$. The resistance mechanisms are known to mainly include overexpression of oncogenic kinases, FLT3 ligand overproduction, bone marrow micro-environment (BME)-mediated protection and acquired resistant mutation ${ }^{[2,7]}$.

Autophagy is an adaptive survival mechanism that is essential for cellular homeostasis in response to various stresses ${ }^{[9]}$. More and more studies ${ }^{[10-12]}$ have linked alteration of autophagy with cancer initiation, progression and treatment resistance, including leukemia, thus autophagy has been shown to be a key therapeutic target. Most recently, Heydt Q et al. ${ }^{[13]}$ reported that FLT3-ITD mutation increased basal autophagy to support leukemic cells survival; also autophagy inhibition overcame FLT3 inhibitor resistance in vitro and vivo, suggesting autophagy might involve in the development and progression of FLT3-ITD mutation AML. However, in FLT3-ITD-positive AML, how autophagy is activated to induce 
resistance to FLT3 inhibitors, and how it mediates the resistance remains unclear. In this study, we mainly explored the correlation of autophagy with FLT3 inhibitor resistance, the inductive effect of acquired mutation and BME on autophagy, and the mechanism of autophagy mediating resistance.

\section{Materials And Methods}

\section{Reagents and antibodies}

Sorafenib and quizartinib (AC220) were purchased from Selleck. Chloroquince (CQ) were bought from Sigma. The antibodies against human-phosphorylated (p)-p44/42 MAPK (ERK1/2, Thr202/Tyr204), pFLT3 (Tyr589/591), p-mTOR (Ser2448), p-S6K (Ser240/244), mTOR, S6K, Beclin-1, LC3B, c-Myc (D84C12) and cleaved caspase-3 (Asp175) were purchased from Cell Signaling Technology. Against ERK2, FLT3 were from Santa Cruz Biotechnology. Anti-GAPHD was purchased from Millipore Sigma.

\section{AML patient samples and FLT3 mutant cell lines}

All patients with FLT3-ITD-positive AML were included from the ClinicalTrials (NCT02474290). The detail of clinical characteristics and treatment protocol had been reported ${ }^{[14]}$. Bone marrow samples were obtained at diagnosis, continued complete response (CCR) or relapsed after obtaining written informed consent from each patient (according to the institutional guidelines of Medicine Institutional Review Boards of Nanfang Hospital, Southern Medical University). The mononuclear (MNC) cells in these samples were purified by Ficoll-Hypaque (Sigma-Aldrich) density gradient centrifugation, and the cells were cultured in RPMI-1640 culture medium supplemented with $10 \%$ fetal calf serum (FCS) prior to treatment.

Mesenchymal stem cells (MSCs), obtained from bone marrow of one of the patients above at leukemia relapse, were cultured at a density of 5,000 cells/cm2 in a-MEM, supplemented with $20 \% \mathrm{FCS}, 1 \% \mathrm{~L}$ glutamine, and $1 \%$ penicillin-streptomycin. The MSCs were used for co-culture experiments after passage.

The Ba/F3-ITD, Ba/F3-D835Y and Ba/F3-ITD+D835Y cell lines, and the human AML cell line MOLM14 were all kindly provided by professor Andreeff Michael (Department of Leukemia Research, Division of Cancer Medicine, The University of Texas MD Anderson Cancer Center, Houston, TX) in 2016. All cell lines were validated by short tandem repeat (STR) DNA fingerprinting using the AmpFISTR Identifiler Kit as described before ${ }^{[15]}$. All cells were maintained in RPMI medium supplemented with $10 \%$ FCS.

\section{Cell viability and apoptosis assays}

Cell viability was assessed using the trypan blue dye exclusion method, and apoptosis was determined via flow cytometry (FACS) by Annexin $\mathrm{V}$ positivity as described ${ }^{[16]}$.

For measuring apoptosis induction in the leukemia cells co-cultured with MSCs, the cells were trypsinized and stained with CD90-PE, CD45-APC and Annexin V-FITC (all from BD Biosciences), and apoptosis was 
assessed by measuring Annexin V-FITC positivity after excluding the CD90+CD45- (used as a MSC marker) cell population.

\section{Transmission Electron Microscopy}

According to our previous report ${ }^{[17]}$, samples were fixed with a solution containing $3 \%$ glutaraldehyde plus $2 \%$ paraformaldehyde in $0.1 \mathrm{M}$ cacodylate buffer, $\mathrm{pH} 7.3$, then washed in $0.1 \mathrm{M}$ sodium cacodylate buffer and treated with $0.1 \%$ Millipore-filtered cacodylate buffered tannic acid, postfixed with $1 \%$ buffered osmium, and stained en bloc with $1 \%$ Millipore-filtered uranyl acetate. The samples were dehydrated in increasing concentrations of ethanol, infiltrated, and embedded in LX-112 medium. The samples were polymerized in a $60^{\circ} \mathrm{C}$ oven for approximately 3 days. Ultrathin sections were cut in a Leica Ultracut microtome (Leica, Deerfield, IL), stained with uranyl acetate and lead citrate in a Leica EM Stainer, and examined in a JEM 1010 transmission electron microscope (JEOL, USA, Inc., Peabody, MA) at an accelerating voltage of $80 \mathrm{kV}$. Micrographs were taken at $7500 \times$ or $50000 \times$ magnification.

\section{Immunoblotting analyses}

The cells were treated with the indicated agents and then collected in lysis buffer. Phosphorylation and total protein levels were determined using Odyssey Infrared Imaging System (LI-COR Biosciences).

\section{Statistical analyses}

The data are presented as the means \pm SD of triplicate samples or assays. The statistical analyses were performed using unpaired Student $t$ test. A 2-sided Fisher exact test was used to determine statistical significance between different groups. A P value $\leq 0.05$ was considered statistically significant.

\section{Results}

\section{FLT3 inhibitor resistant primary FLT3-ITD-positive AML cells showed overexpression of autophagy.}

Six patients with newly diagnosed FLT3-ITD mutant AML, of whom 2 were CCR and 4 relapsed on sorafenib treatment, were included and isolated MNC cells from born marrow at diagnosis and the status of CCR or relapse. The characteristics of the 6 patients were presented in table 1. The primary AML cells being isolated from 2 relapsed patients (Case \#3 with FLT3-ITD mutation and Case \#4 with FLT3ITD+D835Y mutation at relapse) were truly resistant to sorafenib in vitro (figure $1 \mathrm{~A}$ and 1B). Immunoblotting analyses showed increasing expression of LC3B-II in the sorafenibresistant blasts in comparison with the expression before the treatment, in contrast to that, downregulating LC3B-II was found in the sorafenib-sensitive cells (figure 1C).

Table 1. Treatment response and FLT3 mutaions of the 6 patients with FLT3-ITD mutant AML 


\begin{tabular}{|c|c|c|c|}
\hline \multirow[b]{2}{*}{ Case no. } & \multirow{2}{*}{ Response } & \multicolumn{2}{|c|}{ FLT3 mutation } \\
\hline & & Diagnosis & Relapse \\
\hline$\# 1$ & CCR & FLT3-ITD & NA \\
\hline \#2 & CCR & FLT3-ITD & NA \\
\hline \#3 & relapse & FLT3-ITD & FLT3-ITD \\
\hline \#4 & relapse & FLT3-ITD & FLT3-ITD+D835 \\
\hline \#5 & relapse & FLT3-ITD & FLT3-ITD \\
\hline \#6 & relapse & FLT3-ITD & WT \\
\hline
\end{tabular}

CCR, continued complete response; NA, not available; WT, wild type.

\section{Acqured D835Y mutation induced resistance to FLT3 inhibitor and activated autophagy in FLT3-ITD- positive cell lines.}

In order to test activation of autophagy in sorafenib-resistant AML cells, sorafenib-resistant cell lines were built such as Baf3 cells with FLT3-D835Y or FLT3-ITD+D835Y mutation and detected LC3B expression. In line with previous report ${ }^{[18]}, \mathrm{Ba} / \mathrm{F3}-\mathrm{ITD}+\mathrm{D} 835 \mathrm{Y}$ mutant cells were resistant to sorafenib (figure 2A). Compared with $\mathrm{Ba}$ /F3-ITD mutant cells, which was sensitive to sorafenib (figure $2 \mathrm{~A}$ ), both $\mathrm{Ba}$ /F3-D835Y and Ba/F3-ITD+D835Y mutant cells presented higher expression of LC3B-II (figure 2B), indicating that acquired resistant mutation could activate cytoprotective autophagy.

\section{Inhibition of autophagy overcame FLT3 inhibitor resistance in AML with FLT3/ITD mutation.}

The data above showed that sorafenib-resistant leukemia cells expressed higher autophagy, suggesting autophagy activation might be associated with FLT3 inhibitor resistance in FLT-ITD-positive AML. To test this hypothesis, we used CQ to down-regulate autophagy in sorafenib-resistant cells, then detected whether it would strengthen the anti-leukemia effect of sorafenib. As mention above, Ba/F3-ITD+D835Y cells were resistant to sorafenib. After inhibition of autophagy with CQ, Ba/F3-ITD+D835Y cells were turned to be sensitive to sorafenib treatment (figure $3 A$ ). In line with this, western blot showed sorafenib increased the expression of cleaved-caspase 3 in Ba/F3-ITD+D835Y cells after being dealt with CQ versus did not without $\mathrm{CQ}$ co-treatment (figure $3 \mathrm{~B}$ ). In addition, inhibition of autophagy with $\mathrm{CQ}$ also enhanced the anti-leukemia effect of sorafenib in Ba/F3-ITD cells (figure 3A and 3B). Furthermore, sorafenib-resistant primary AML cells with FLT3-ITD mutation (case \#3) or FLT3-ITD+D835Y mutation (case \#4) were also sensitized to sorafenib after being dealt with CQ (figure 3C and 3D). Taking together, sorafenib-resistant leukemia cells overexpressed autophagy; inhibition of autophagy partly overcame sorafenib resistance, suggesting activation of autophagy could be an important factor for FLT3 inhibitor resistance in FLT3-ITD mutated AML cells. 


\section{Inhibition of autophagy enhanced the suppression efficacy of FLT3 inhibitor on FLT3 downstream signaling.}

We then went further to explore how sorafenib worked after autophagy was inhibited. Immunoblotting data showed that, in Ba/F3-ITD+D835Y cells, without CQ co-treatment, sorafenib could only downregulate the expression of p-FLT3, but not FLT3 downstream signaling, and no pro-apoptotic affect was induced. However, after being dealt with $\mathrm{CQ}$, autophagy was inhibited, sorafenib significantly suppressed the downstream signaling of FLT3 and induced the expression of cleaved caspase-3 (figure 4), which accorded with autophagy inhibition enhancing the killing effect of sorafenib in Ba/F3-ITD+D835Y cells, indicating autophagy might bypass activate FLT3 downstream signaling to decrease the cytotoxic effect of FLT3 inhibitor in FLT3 inhibitor resistant leukemic cells.

\section{Born marrow micro-environment mediated FLT3 inhibitor resistance in FLT3-ITD mutant cells via activating autophagy.}

It is reported that BME could up-regulate autophagy to mediate chemotherapy resistance in AML ${ }^{[19]}$. Whether BME-mediated autophagy could induce FLT3 inhibitor resistance in FLT3-ITD-positive AML remains unsure. To test the affect of BME on activation of autophagy and mediation of FLT3 inhibitor resistance in FLT3-ITD mutant cells, we detected, respectively, the expression of autophagy and the antileukemia effect of FLT3 inhibitors including sorafenib and AC220 in FLT3-ITD-mutated cells with versus without MSCs co-culture, and incubated in hypoxia versus normoxia.

After co-culture with MSCs, the number of phagosomes in MOLM14 cells being detected with transmission electron microscopy showed significant increase (figure 5A). In accordance with this, the expression of LC3B-II and Beclin-1 with western blot assessment also up-regulated with MSCs coculture versus without (figure 5B). Meanwhile, in support of this, hypoxia also stimulated autophagy, presenting as higher expression of LC3B-II and Beclin-1 in MOLM14 cells after being incubated in hypoxia versus normoxia (figure $5 B$ ).

In addition, MSCs significantly decreased the killing effect of sorafenib either at the concentration of $40 \mathrm{nM}$ or $80 \mathrm{nM}$ in MOLM14 cells (figure 6A). The same was found in the treatment of AC220 (figure 7B). In agreement with this, the immunoblotting data showed that in contrast to the result without MSCs coculture, MSCs upregulated the expression of LC3B-II and Beclin-1 in MOLM14 cells and weakened the inhibition efficacy of sorafenib on FLT3 signaling pathway, especially on FLT3 downstream signaling, presenting as reducing suppression on $\mathrm{p}-\mathrm{FLT} 3$, $\mathrm{p}$-ERK1/2 and p-mTOR with MSCs co-culture, and then decreased the expression of cleaved-caspase 3, which could explain MSCs decreased the anti-leukemia effect of sorafenib in MOLM14 cells, suggesting that MSCs-mediated FLT3 inhibitor resistance in FLT3ITD mutant cells might be associated with activating autophagy.

Then we used CQ to inhibit autophagy in MOLM14 cells. After inhibition of autophagy, MSCs-mediated sorafenib or AC220 resistance was overcome (figure 7A and 7B), and even the anti-leukemia effect of sorafenib or AC220 was sensitized regardless of MSCs co-culture (figure 7A and 7B), in agreement with 
the report of FLT3-ITD up-regulating autophagy to mediate resistance to FLT3 inhibitors in AML ${ }^{[13]}$. Taking together, BME mediated FLT3 inhibitor resistance via up-regulation of autophagy, inhibition of autophagy could overcome BME-mediated FLT3 inhibitor resistance, in FLT3-ITD-positive AML.

\section{Discussion}

FLT3 inhibitor resistance is the important reason for leukemia relapse in FLT3-ITD mutation AML ${ }^{[2,7-8]}$. In the present study, we revealed, in FLT3-ITD mutation AML, FLT3 inhibitor resistant cells overexpressed autophagy; Acquired D835Y mutation or BME activated autophagy to mediate FLT3 inhibitor resistance; Autophagy activation reduced the suppression efficacy of FLT3 inhibitor on FLT3 downstream signaling and then decreased their pro-apoptotic effect; Inhibition of autophagy enhanced the anti-leukemia effect of FLT3 inhibitors, overcame FLT3 inhibitor resistance, mediated by either acquired mutation or BME. Our data further supports that autophagy significantly involves in leukemia progression and resistance, could be a promising therapeutic target in FLT3-ITD-positive AML.

Our results accord with previous work ${ }^{[10-13,20-21]}$, showing that autophagy is closely associated with resistance in AML, especially in the patients with FLT3-ITD mutation. Studies ${ }^{[17,20-21]}$ demonstrate that autophagy activation may promote survival of leukemia cells and induce resistance to chemo or target therapies. Activation of cytoprotective autophagy is found in cytarabine-resistant AML cells, in which

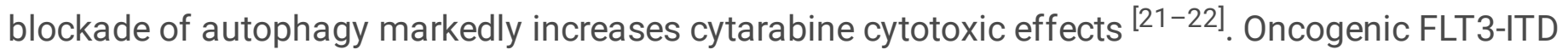
increases autophagic flux to support AML cell survival and proliferation. Inhibition of autophagy overcomes FLT3 inhibitor resistance in FLT3-ITD-positive cells ${ }^{[13,23]}$. In our study, FLT3 inhibitor resistant leukemia cells showed significantly activating autophagy as compared with before the treatment or FLT3 inhibitor sensitive cells. In agreement with the reports above ${ }^{[13,23]}$, autophagy inhibition sensitized those resistant cells to FLT3 inhibitors.

In this study, acquired D835Y mutation and BME were the two important factors for stimulation of autophagy in FLT3-ITD-positive cells. As it is known that acquired mutation is the key factor for resistance to FLT3 inhibitors in FLT3 mutated AML ${ }^{[2,7-8]}$. For the resistance mechanism, except for known changing molecular conformation ${ }^{[24]}$, to our knowledge, we are the first to reveal its association with autophagy activation, showing as higher expression of autophagy in Ba/F3-ITD + D835Y or Ba/F3D835Y versus Ba/F3-ITD cells. Inhibition of autophagy with CQ enhanced the anti-leukemia effect of sorafenib in not only leukemia cell lines but also primary leukemic blasts with FLT3-ITD + D835Y mutation, which opens a window for overcoming FLT3 inhibitor resistance to AML with acquired D835 mutation. In addition, secondary mutation is known to be acquired under clone selective pressure [25]. Autophagy is an adaptive survival mechanism for leukemia cells in response to various stresses ${ }^{[9-10,12]}$. The question of the causal link between acquired mutation and autophagy activation remains open. In line with stimulation of cytoprotective autophagy against thecytarabine/anthracycline combination by BME ${ }^{[19]}$, we also observed BME induced cytoprotective autophagy against FLT3 inhibitors, which could be overcome by autophagy inhibition. The way of BME stimulating autophagy needs further research. 
It is universally acknowledged that autophagy exerts complicated affects on the progression of cancers including AML ${ }^{[11-12]}$. Accumulating evidence ${ }^{[26-28]}$ has shown that autophagy activation, triggers the selective elimination of impaired or extra intracellular contents, such as mitophagy, then supports the maintenance and self-renewal capacity of cancer stem cells, acting as a regulatory or cytoprotective adaptive mechanism, leading to malignant progression of different types of cancers including AML. In FLT3-ITD-positive AML, FLT3-ITD mutation increases basal autophagy to support leukemic cell survival and proliferation via transcription factor ATF4 (activating transcription factor 4). Inhibition of autophagy or ATF4 enhances the anti-leukemia effect of FLT3 inhibitors ${ }^{[13]}$. Yet, how autophagy mediating FLT3 inhibitor resistance remains unclear. In our study, we found that sorafenib along could only suppress the p-FLT3 level but not the FLT3 downstream signaling and neither induce pro-apoptotic effect in Ba/F3-ITD + D835Y cells. However, after inhibition of autophagy, sorafenib markedly suppressed not only p-FLT3 but also FLT3 downstream signaling, and finally promoted cell death. In accordance with that, stimulation of autophagy with MSCs also weakened the inhibitory activity of sorafenib on FLT3 pathway, especially on FLT3 downstream signaling, and then decreased its anti-leukemia effect. In contrast, inhibition of autophagy overcame MSCs-mediated FLT3 inhibitor resistance. Taking together, it suggests that autophagy activation might bypass activate FLT3 downstream signaling to eliminate the suppressive effect of FLT3 inhibitors on FLT3 pathway, which calls further study to explore the mechanism of bypass activation of FLT3 downstream with autophagy.

More and more studies have demonstrated autophagy could be a promising therapeutic target for overcoming drug resistance in AML ${ }^{[11-13,19-20]}$. As mention above, conventional chemotherapeutic agents including cytarabine and daunorubicin are able to induce autophagy as a survival mechanism to resist cytotoxic stress and counteract their therapeutic effects; inhibition of autophagy could synergize with these agents to overcome drug resistance, improve clinical outcomes in AML therapy ${ }^{[12,19,29]}$. Apart from chemotherapy, in targeting treatment, such as BET inhibitors ${ }^{[20]}$, histone methyltransferase inhibitors ${ }^{[30]}$, BCL2 inhibitors ${ }^{[31]}$, and FLT3 inhibitors reported in the present study, could also activate autophagy, serving as a cytoprotective adaptive mechanism against clone selective stress, to contribute to the drug resistance of AML stem cells; co-targeting autophagy could enhance the anti-leukemia effect of these inhibitors. Moreover, $\mathrm{BME}$ is found to be another important factor to stimulate autophagy to mediate resistance to conventional chemotherapy ${ }^{[19]}$, also targeting therapy as FLT3 inhibitors presented in this study; inhibition of autophagy could also eliminate BME-mediated resistance. In the FLT3-ITDpositive AML, combination of FLT3 inhibitor quizartinib with autophagy inhibitor Lys05 markedly improved proliferation inhibition and apoptosis induction in comparison with quizartinib alone [23]. Autophagy suppression by PIK3C3/VPS34 (phosphatidylinositol 3-kinase catalytic subunit type 3) inhibitor SAR405 or shRNA against ATG12 overcame the resistance of MOLM-14 cells with FLT3-D835Y mutation to quizartinib in vitro and vivo ${ }^{[13]}$. In this study, autophagy was inhibited with $\mathrm{CQ}$, as a result, in FLT3-ITD-positive cell lines and primary AML blasts, the acquired resistance to FLT3 inhibitors, from either acquired M835Y mutation or BME, was remarkably overcome. All of these further support autophagy should be a critical target in AML therapy, especially in overcoming drug resistance. 


\section{Conclusion}

Our findings demonstrate autophagy activation is closely associated with FLT3 inhibitor resistance in FLT3-ITD-positive AML. Autophagy might be stimulated by acquired mutation or BME, and then decrease the anti-leukemia effect of FLT3 inhibitors via bypass activation of FLT3 downstream signaling. Targeting autophagy could be a promising strategy to overcome FLT3 inhibitor resistance.

\section{Abbreviations}

FLT3-ITD: Fms-like tyrosine kinase 3-internal tandem duplication; AML: acute myeloid leukaemia; BME: bone marrow micro-environment; CQ: chloroquince; CCR: continued complete response; MNC: mononuclear; FCS: fetal calf serum; MSCs: Mesenchymal stem cells; STR: short tandem repeat; FACS: flow cytometry; SD: standard deviation; BET: bromodomain and extra-terminal; BCL2: B-cell lymphoma 2; ATG12: autophagy-related gene 12.

\section{Declarations}

\section{Acknowledgements}

We would like to thank professor Andreeff Michael providing us FLT3 mutation leukemic cell lines.

\section{Authors' contributions}

G.Y. and D.X. designed the experiments. D.X., Z.Y., Y.Y. and C.H. conducted the

experiments. D.X., G.Y, X.J. and Q.L. were in charge of clinical data and patient sample collection. Y.C., Q.W., L.J., C.Y. and G.Y. analyzed the data. D.X. wrote the manuscript. All authors read and approved the final manuscript.

\section{Funding}

This work was supported by the Natural Science Foundation of Guangdong Province, China (no. 2020A1515011514 and no. 2021A1515012103) and the Cancer Research Foundation of Shiyao-CSCO (no. Y-SY201902-0052).

\section{Availability of data and materials}

All data generated or analyzed during this study are included in this manuscript.

\section{Ethics approval and consent to participate}

The clinical samples were collected from patients after obtaining written informed consent from each patient (according to the institutional guidelines of Medicine Institutional Review Boards of Nanfang Hospital, Southern Medical University). 


\section{Consent for publication}

Not applicable.

\section{Competing interests}

The authors have no competing interest to declare.

\section{Author details}

All authors are from Department of Hematology, Nanfang Hospital, Southern Medical University, No.1838 Guangzhou Avenue North, Guangzhou 510515, China.

\section{References}

1. Papaemmanuil E, Gerstung M, Bullinger L, Gaidzik VI, Paschka P, Roberts ND, et al. Genomic classification and prognosis in acute myeloid leukemia. N Engl J Med. 2016; 374: 2209-21.

2. Antar Al, Otrock ZK, Jabbour E, Mohty M, Bazarbachi A. FLT3 inhibitors in acute myeloid leukemia: ten frequently asked questions. Leukemia. 2020; 34: 682-96.

3. Bazarbachi A, Bug G, Baron F, Brissot E, Ciceri F, Dalle IA, et al. Clinical practice recommendation on hematopoietic stem cell transplantation for acute myeloid leukemia patients with FLT3 internal tandem duplication: a position statement from the Acute Leukemia Working Party of the European Society for Blood and Marrow Transplantation. Haematologica. 2020; 105: 1507-16.

4. Xuan L, Wang Y, Huang F, Jiang E, Deng L, Wu B, et al. Effect of sorafenib on the outcomes of patients with FLT3-ITD acute myeloid leukemia undergoing allogeneic hematopoietic stem cell transplantation. Cancer. 2018; 124: 1954-63.

5. Mark L. Midostaurin approved for FLT3-mutated AML. Blood. 2017; 129(26): 3403-6.

6. Rollig C, Serve H, Huttmann A, Noppeney R, Müller-Tidow C, Krug U, et al. Addition of sorafenib versus placebo to standard therapy in patients aged 60 years or younger with newly diagnosed acute myeloid leukaemia (SORAML): a multicentre, phase 2, randomised controlled trial. Lancet Oncol. 2015; 16: 1691-9.

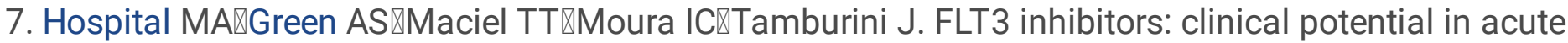
myeloid leukemia. Onco Targets Ther. 2017; 10: 607-15.

8. Leung AYH, Man CH, Kwong YL. FLT3 inhibition: a moving and evolving target in acute myeloid leukaemia. Leukemia. 2013; 27: 260-8.

9. Mehrpour M, Esclatine A, Beau I, Codogno P. Overview of macroautophagy regulation in mammalian cells. Cell Res. 2010; 20(7): 748-62.

10. Patrick A, Alexandre P. Autophagy, a key mechanism of oncogenesis and resistance in leukemia. Blood. 2017; 129(5): 547-52. 
11. Amaravadi RK, Kimmelman AC, Debnath J. Targeting autophagy in cancer: recent advances and future directions. Cancer Discov. 2019; 9(9): 1167-81.

12. Du W, Xu A, Huang Y, Cao J, Zhu H, Yang B, et al. The role of autophagy in targeted therapy for acute myeloid leukemia. Autophagy. 2020 Sep 22; DOI: 10.1080/15548627.2020.1822628

13. Heydt Q, Larrue C, Saland E, Bertoli S, Sarry JE, Besson A, et al. Oncogenic FLT3-ITD supports autophagy via ATF4 in acute myeloid leukemia. Oncogene. 2018; 37: 787-97.

14. Xuan L, Wang Y, Huang F, Fan ZP, Xu YJ, Sun j, et al. Sorafenib maintenance in patients with FLT3ITD acute myeloid leukaemia undergoing allogeneic haematopoietic stem-cell transplantation: an open-label, multicentre, randomised phase 3 trial. Lancet Oncol. 2020; 21(9): 1201-12.

15. Zhang W, Borthakur G, Gao C, Chen Y, Mu H, Ruvolo VR, et al. The dual MEK/FLT3 inhibitor E6201 exerts cytotoxic activity against acute myeloid leukemia cells harboring resistance-conferring FLT3 mutations. Cancer Res. 2016; 76(6): 1528-37.

16. Clodi K, Kliche KO, Zhao S, Weidner D, Schenk T, Consoli U, et al. Cell surface exposure of phosphatidylserine correlates with the stage of fludarabine-induced apoptosis in chronic lymphocytic leukemia (CLL) and expression of apoptosis-regulating genes. Cytometry. 2000; 40: 19-25.

17. Lu Z, Xu N, He B, Pan C, Lan Y, Zhou H, Liu X. Inhibition of autophagy enhances tigecycline's selective anti-cancer activity to overcome drug resistance of chronic myeloid leukemia. J Exp Clin Cancer Res. 2017; 36(1): 43.

18. Zhang W, Konopleva M, Shi YX, McQueen T, Harris D, Ling X, et al. Mutant FLT3: a direct target of sorafenib in acute myelogenous leukemia. J Natl Cancer Inst. 2008; 100(3): 184-98.

19. Piya S, Kornblau SM, Ruvolo VR, Mu H, Ruvolo PP, McQueen T, et al. Atg7 suppression enhances chemotherapeutic agent sensitivity and overcomes stroma-mediated chemoresistance in acute myeloid leukemia. Blood. 2016; 128(9): 1260-9.

20. Jang JE, Eom JI, Jeung HK, Cheong JW, Lee JY, Kim JS, et al. AMPK-ULK1-Mediated autophagy confers resistance to BET inhibitor JQ1 in acute myeloid leukemia Stem Cells. Clin Cancer Res. 2017; 23(11): 2781-94.

21. Sumitomo Y, Koya J, Nakazaki K, Kataoka K, Tsuruta-Kishino T, Morita K, et al. Cytoprotective autophagy maintains leukemia-initiating cells in murine myeloid leukemia. Blood. 2016; 128(12): 1614-24.

22. Kim Y, Eom JI, Jeung HK, Jang JE, Kim JS, Cheong JW, et al. Induction of cytosine arabinosideresistant human myeloid leukemia cell death through autophagy regulation by hydroxychloroquine. Biomed Pharmacother. 2015; 73: 87-96.

23. Qiu S, Yan C, Paterson AJ, Li H, Bhatia R. Role of enhanced autophagy in resistance of FLT3-ITD AML stem cells to FLT3 TKI treatment. Blood. 2018 Nov 29; 132(Suppl_1): 1358-1358. DOI: 10.1182/blood-2018-99-115702

24. Smith CC, Lin K, Stecula A, Sali A, Shah NP. FLT3 D835 Mutations Confer Differential Resistance to Type II FLT3 Inhibitors. Leukemia. 2015; 29(12): 2390-2. 
25. Moore AS, Faisal A, Gonzalez de Castro D, Bavetsias V, Sun C, Atrash B, et al. Selective FLT3 inhibition of FLT3-ITD+ acute myeloid leukaemia resulting in secondary D835Y mutation: a model for emerging clinical resistance patterns. Leukemia. 2012; 26: 1462-70.

26. Nazio F, Bordi M, Cianfanelli V, Locatelli F, Cecconi F. Autophagy and cancer stem cells: molecular mechanisms and therapeutic applications. Cell Death and Differentiation. 2019; 26(4): 690-702.

27. Nguyen TD, Shaid S, Vakhrusheva O, Koschade SE, Klann K, Thölken M, et al. Loss of the selective autophagy receptor p62 impairs murine myeloid leukemia progression and mitophagy. Blood. 2019; 133(2): 168-79.

28. Pei S, Minhajuddin M, Adane B, Khan N, Stevens BM, Mack SC, et al. AMPK/FIS1-mediated mitophagy is required for self-renewal of human AML stem cells. Cell Stem Cell. 2018; 23(1): 86-100. e6.

29. Putyrski M, Vakhrusheva O, Bonn F, Guntur S, Vorobyov A, Brandts C, et al. Disrupting the LC3 Interaction Region (LIR) Binding of Selective Autophagy Receptors Sensitizes AML Cell Lines to Cytarabine. Front Cell Dev Biol. 2020; 8: 208. doi: 10.3389/fcell.2020.00208

30. Jang JE, Eom JI, Jeung HK, Chung H, Kim YR, Kim JH, et al. PERK/NRF2 and autophagy form a resistance mechanism against G9a inhibition in leukemia stem cells. J Exp Clin Canc Res. 2020; 39(1). DOI: 10.1186/s13046-020-01565-3

31. Folkerts H, Wierenga AT, van den Heuvel FA, Woldhuis RR, Kluit DS, Jaques J, et al. Elevated VMP1 expression in acute myeloid leukemia amplifies autophagy and is protective against venetoclaxinduced apoptosis. Cell Death Dis. 2019; 10(6): 421.

\section{Figures}


A

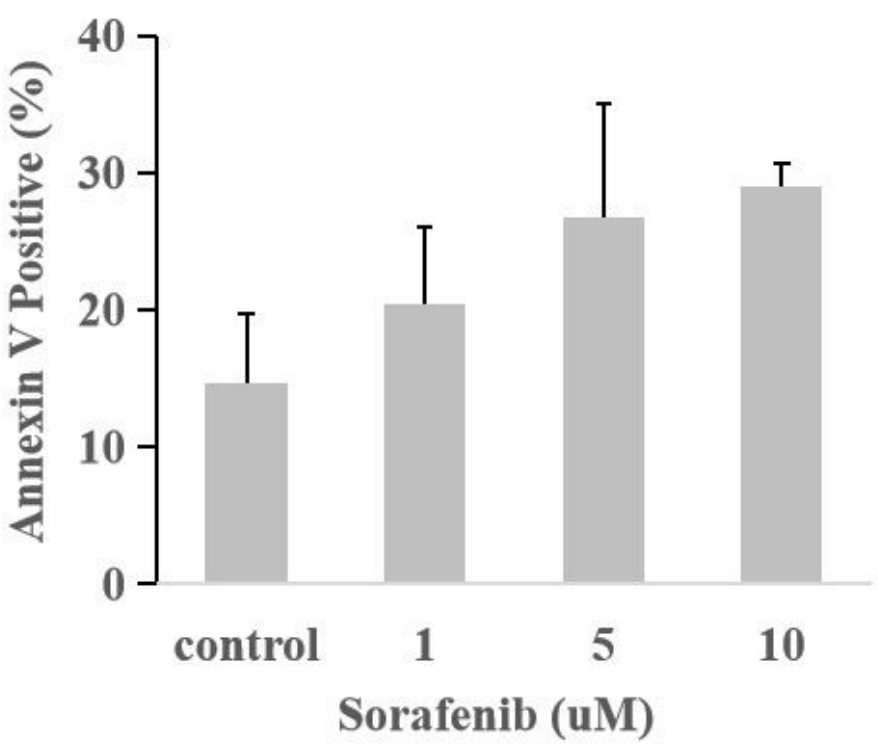

B Ca \#4 FLT3-ITD+D835Y

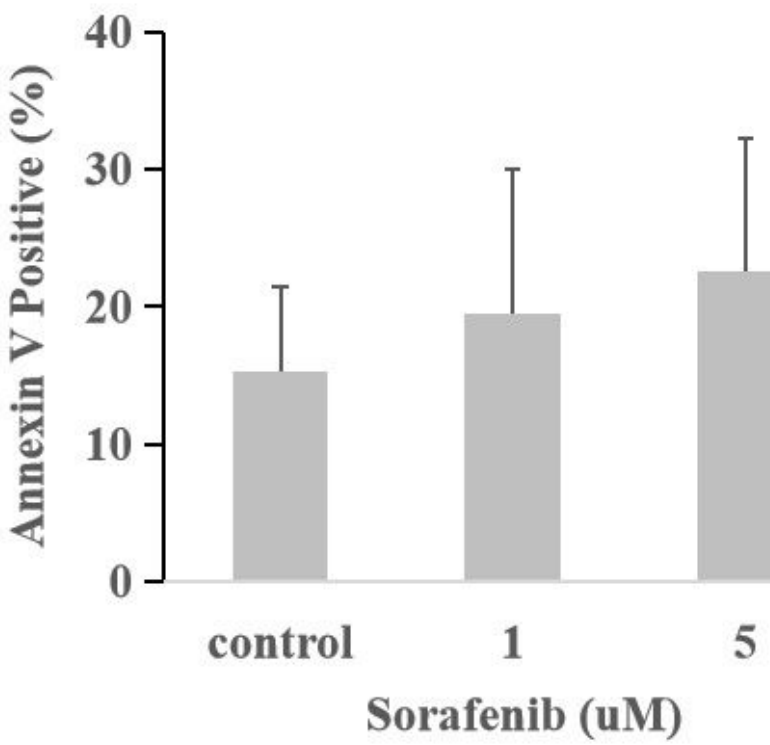

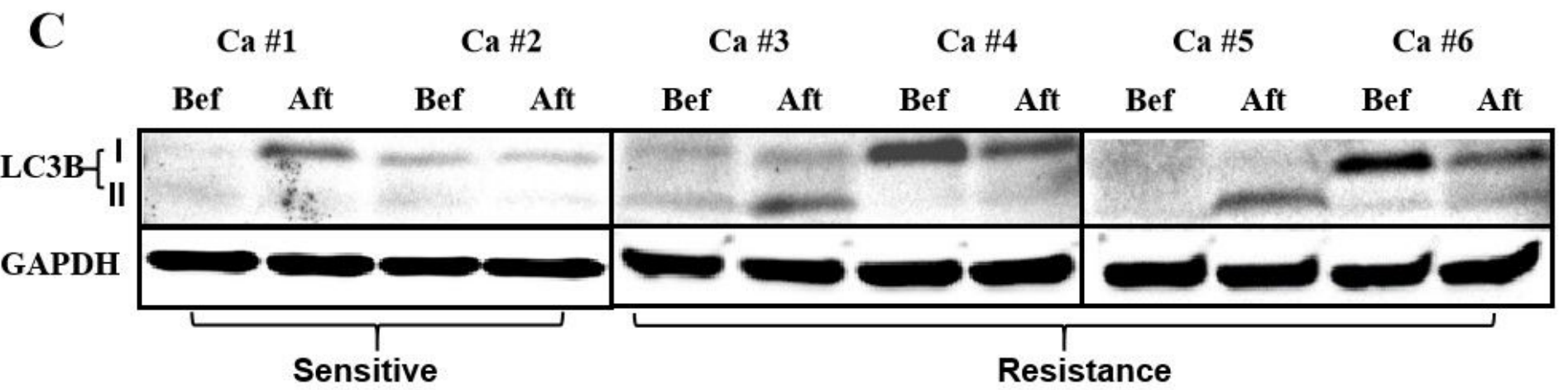

\section{Figure 1}

Sorafenib resistant primary AML cells showed overexpression of autophagy. (A) Primary AML blasts with FLT3-ITD mutation from the patient (case no. 3) who relapsed on sorafenib maintenance therapy were resistant to sorafenib. (B) Primary AML blasts with FLT3-ITD+D835Y mutation from the patient (case no. 4) who relapsed on sorafenib maintenance therapy were resistant to sorafenib. (C) Sorafenib sensitive primary AML cells from the patients with FLT3-ITD mutation who were continued complete response (CCR) with sorafenib therapy showed decreasing expression of LC3B-Il after the treatment of sorafenib as compared with before the treatment. In contrast to the sorafenib sensitive cells, sorafenib resistant AML cells from the patients who relapsed on sorafenib therapy showed increasing expression of LC3B-II after relapse in comparison with before the treatment. 
A

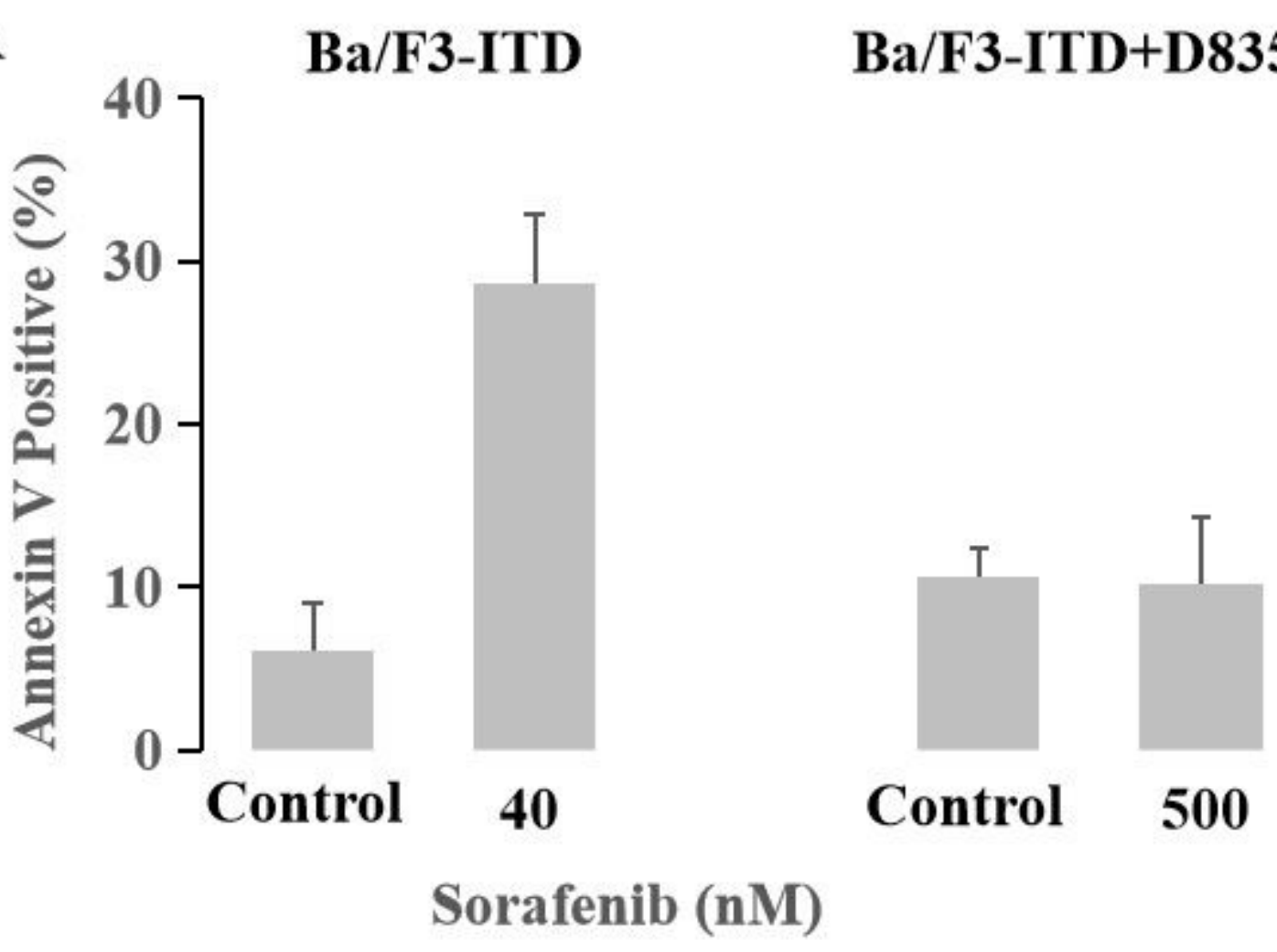

B

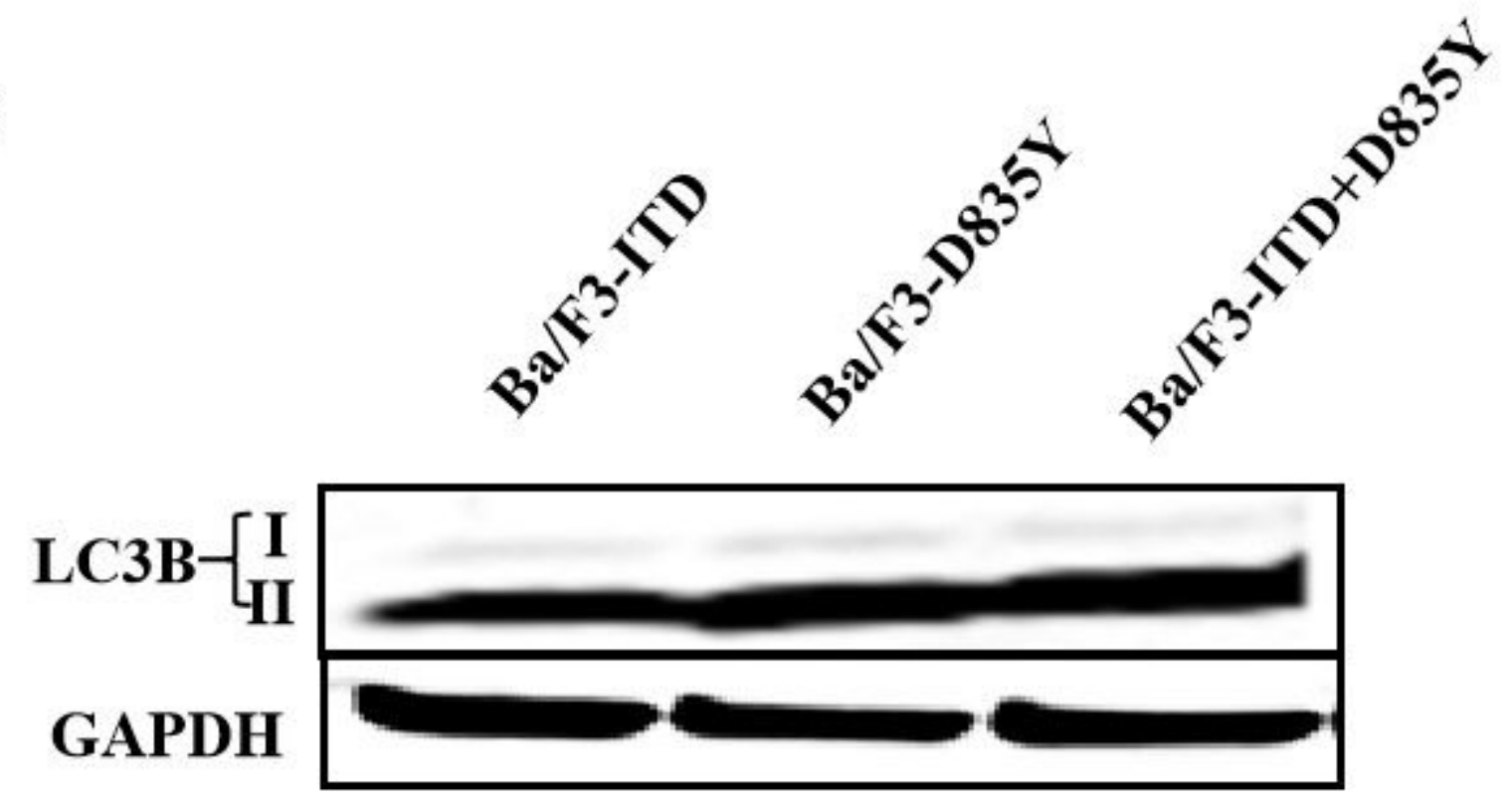

Figure 2

Acquired D835Y mutation induced sorafenib resistance and activated autophagy in FLT3-ITD-positive cell lines. (A) Ba/F3-ITD cell line was sensitive to sorafenib treatment with the apoptosis rate of $29.0 \pm 3.1 \%$ at the concentration of $40 \mathrm{nM}$. After acquired D835Y mutation, Ba/F3-ITD+D835Y cell line showed resistant to sorafenib with the apoptosis rate of $10.5 \pm 3.0 \%$ at the concentration of $500 \mathrm{nM}$. (B) In comparison with $\mathrm{Ba} / \mathrm{F3}$-ITD cells, both Ba/F3-D835Y and Ba/F3-ITD+D835Y cells, which were resistant to sorafenib, showed increasing expression of LC3B-II. 
A

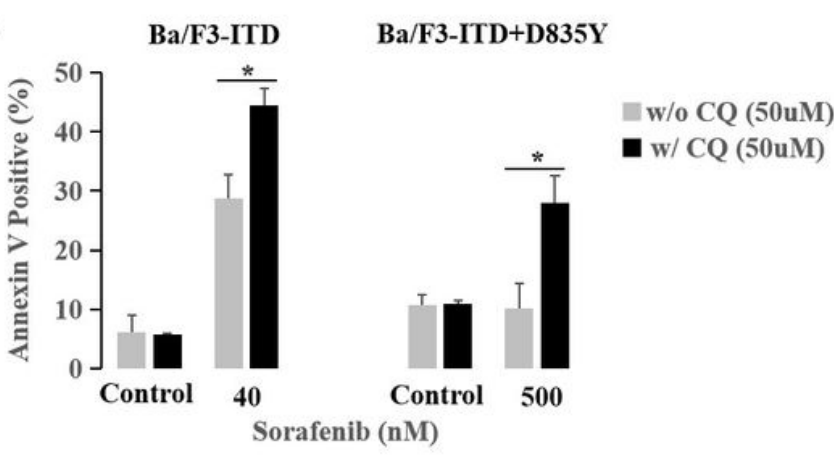

C

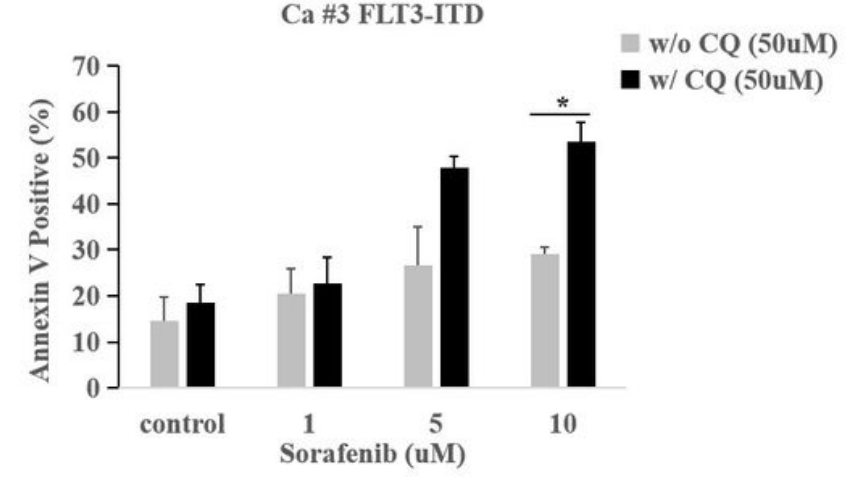

B

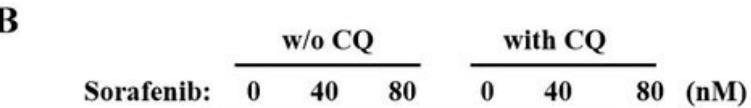
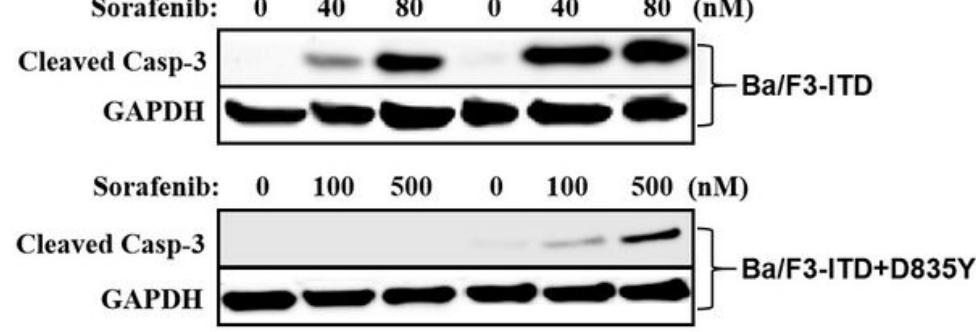

D

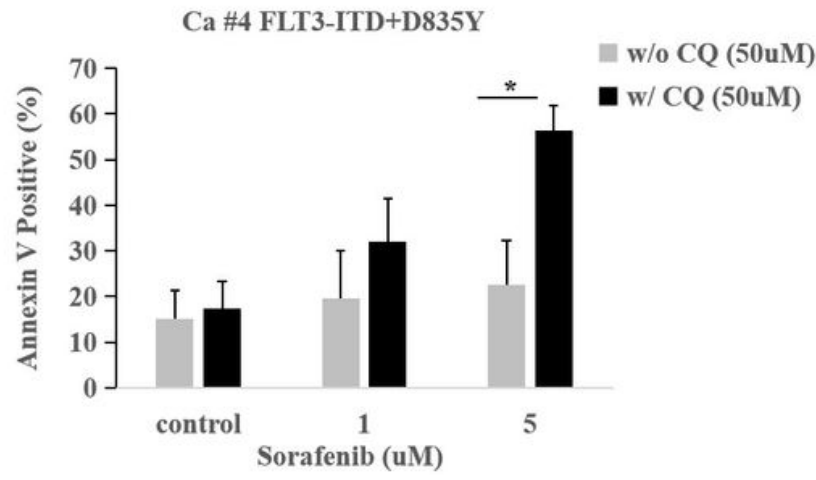

\section{Figure 3}

Inhibition of autophagy enhanced anti-leukemia effect of sorafenib in FLT3-mutated leukemia cells. (A) Inhibition of autophagy with CQ, the anti-leukemia effect of sorafenib in both Ba/F3-ITD (w/o CQ vs. w/ CQ, $29.0 \pm 3.1 \%$ vs. $44.5 \pm 2.2 \%, P=0.038$ ) and Ba/F3-ITD+D835Y cells (w/o CQ vs. w/ CQ, 10.0 $\pm 3.0 \%$ vs. $28.7 \pm 3.6 \%, P=0.041$ ) was significantly enhanced. (B) Western blot showed sorafenib markedly increased the expression of cleaved caspase 3 in both Ba/F3-ITD and Ba/F3-ITD+D835Y cells after being dealt with CQ versus without CQ. (C) CQ enhanced the anti-leukemia effect of sorafenib in sorafenib-resistant primary AML cells with FLT3-ITD mutation (w/o CQ vs. w/ CQ, sorafenib at 5uM, 26.7 $28.2 \%$ vs.

$47.8 \pm 2.7 \%, P=0.075$; sorafenib at $10 u M, 29.0 \pm 1.6 \%$ vs. $53.5 \pm 4.3 \%, P=0.018)$. (D) $C Q$ enhanced the antileukemia effect of sorafenib in sorafenib-resistant primary AML cells with FLT3-ITD+D835Y mutation (w/o CQ vs. $w / C Q$, sorafenib at $5 u M, 22.6 \pm 9.6 \%$ vs. $56.5 \pm 5.4 \%, P=0.049$ ). 


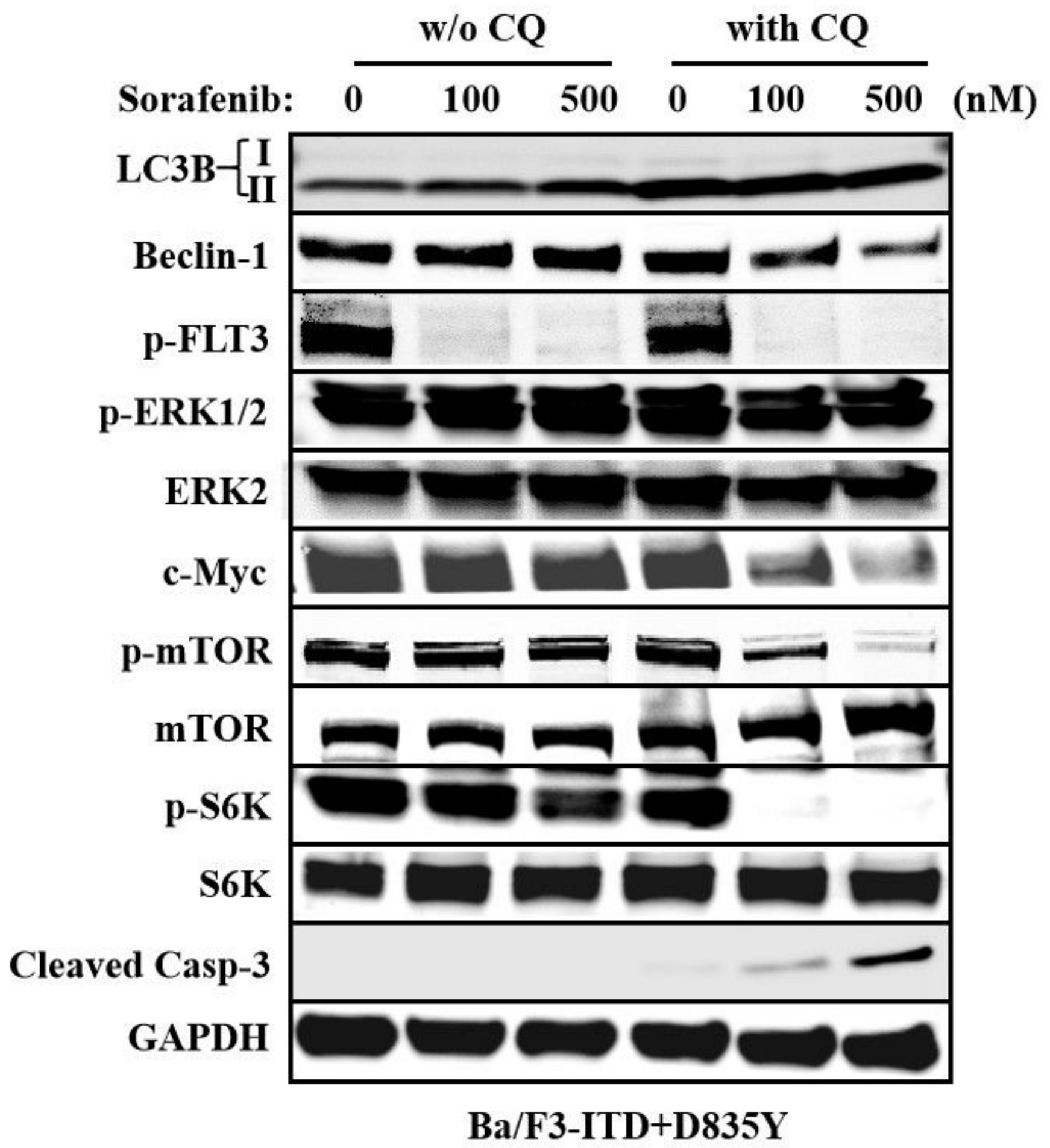

Figure 4

Inhibition of autophagy enhanced suppression efficacy of sorafenib on FLT3 downstream signaling and pro-apoptotic affect in Ba/F3-ITD+D835 mutated cells. In Ba/F3-ITD+D835Y cells, without CQ cotreatment, sorafenib could only down-regulate the expression of p-FLT3, but not FLT3 downstream signaling, and no cleaved caspase- 3 was induced. After being dealt with $C Q$, autophagy was inhibited, sorafenib significantly suppressed the downstream signaling of FLT3 and up-regulated cleaved caspase3. 
A

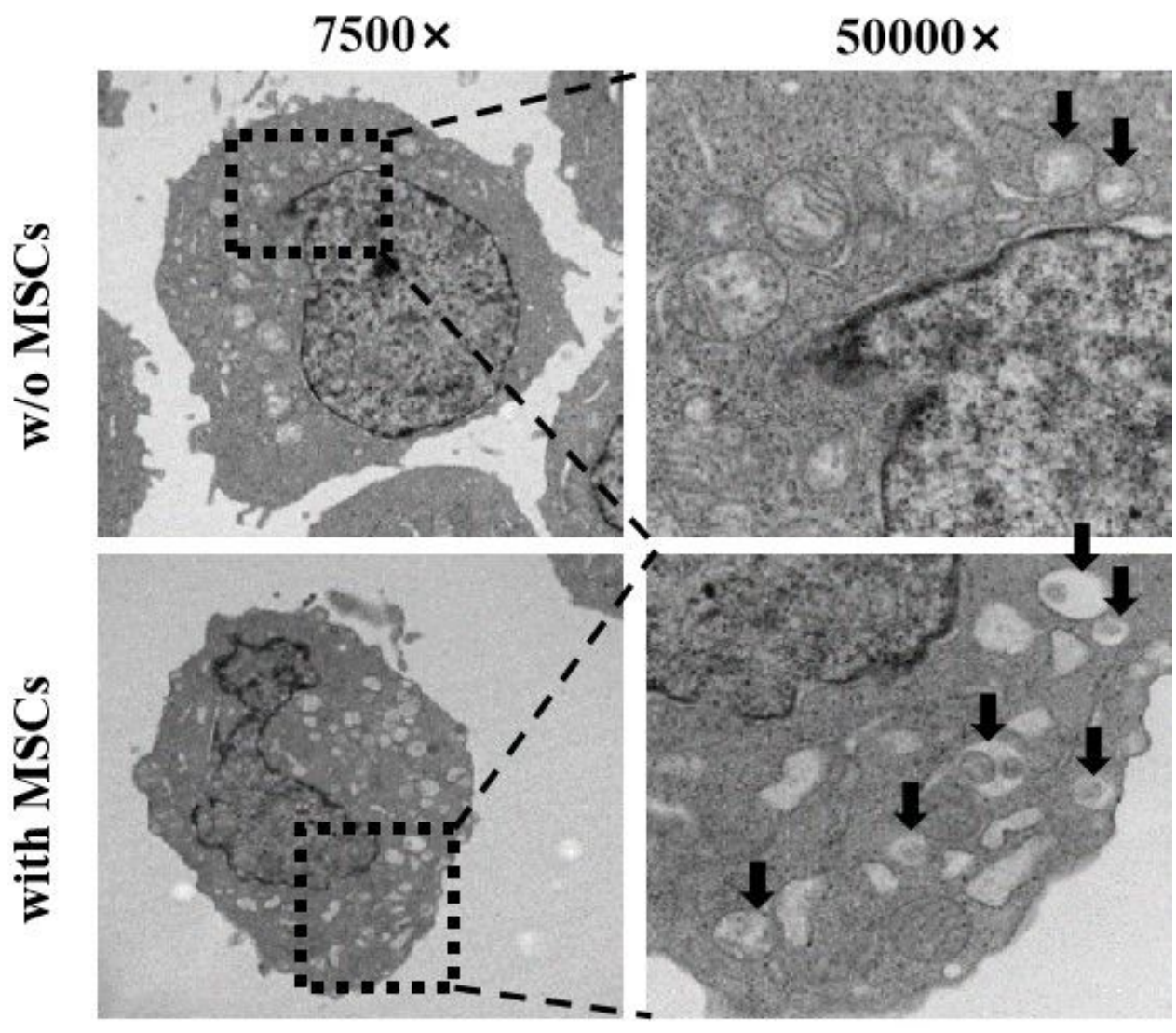

B

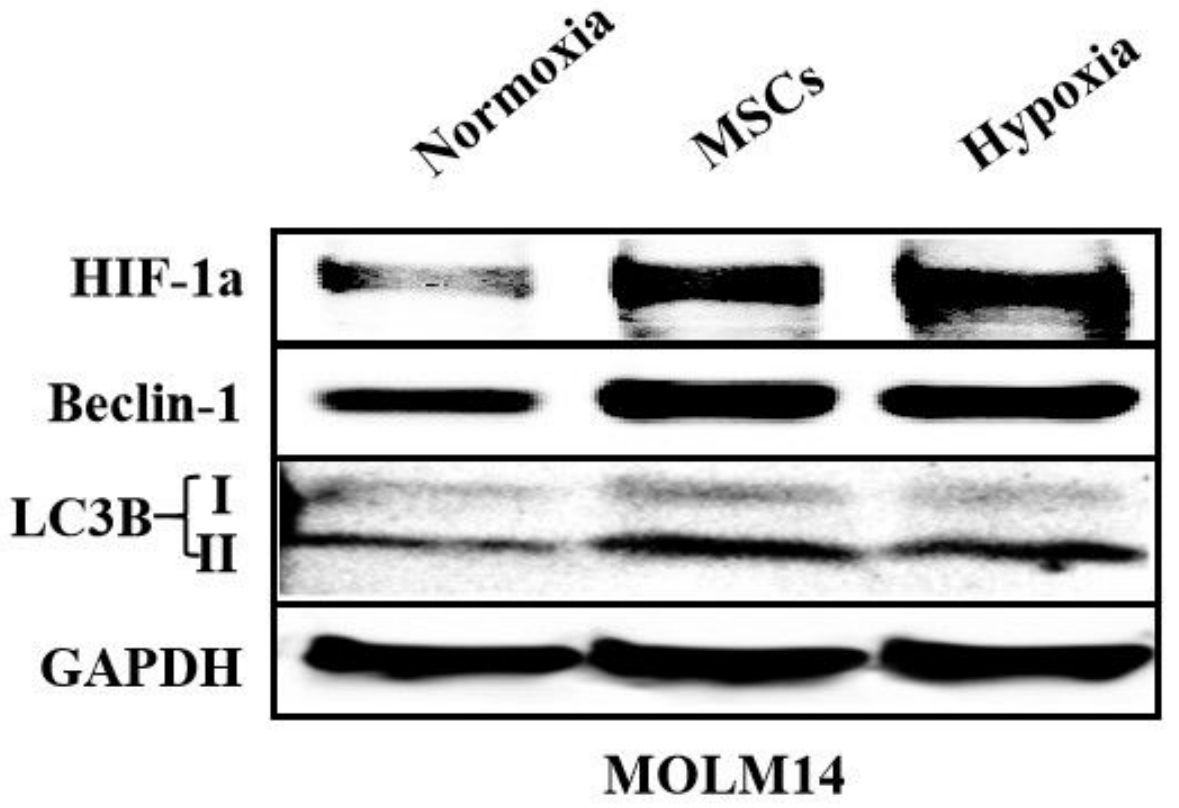

Figure 5

Co-culture with MSCs and hypoxia up-regulated autophagy in AML cells with FLT3-ITD mutation. (A) Versus without MSCs co-culture, MSCs significantly increased the number of phagosomes (under the arrows) in MOLM14 cells being detected with transmission electron microscopy. (B) Immunoblotting analyse showed MSCs and hypoxia increased the expression of LC3B-II and Beclin-1 in MOLM14 cells. 


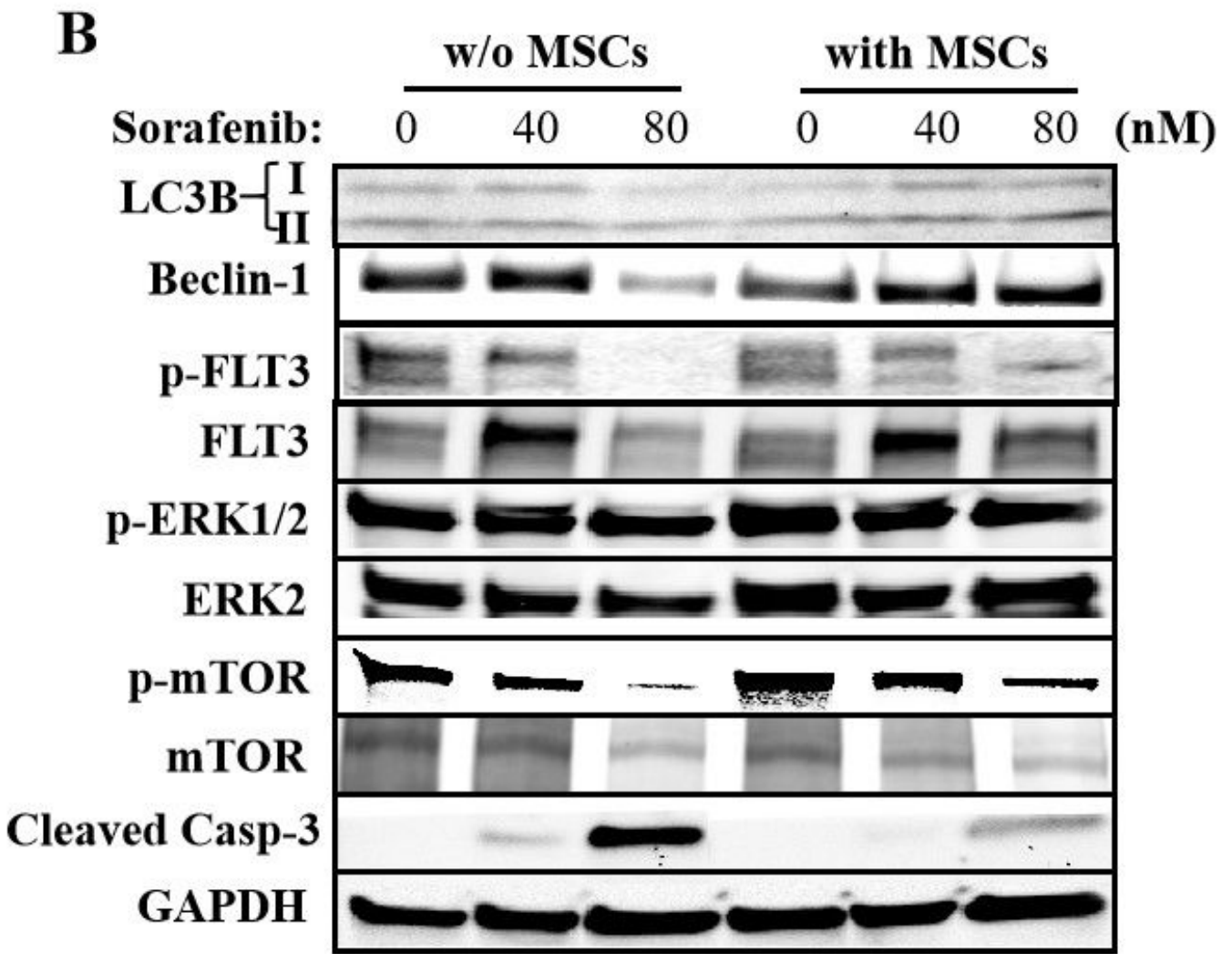

Figure 6

MSCs decreased the anti-leukemia effect of sorafenib via upregulation of autophagy in FLT3-ITD-positive AML cells. (A) After co-culture with MSCs, the killing effect of sorafenib in MOLM14 cells decreased significantly, showing as the apoptosis rate of $22.0 \% \pm 2.1 \%$ vs. $41.2 \% \pm 2.5 \%$ without MSCs co-culture $(P=0.014)$ at $40 \mathrm{nM}$ and $34.8 \% \pm 3.0 \%$ vs. $61.9 \% \pm 1.9 \%(P=0.008)$ at $80 \mathrm{nM}$, respectively. (B)

Immunoblotting analyse showed that, versus without MSCs co-culture, MSCs up-regulated the expression 
of LC3B-II and Beclin-1 and remarkably decreased the inhibition efficacy of sorafenib on FLT3 pathway, especially on FLT3 downstream signaling, presenting as reducing suppression on p-FLT3, p-ERK1/2 and p-mTOR with MSCs co-culture, and then decreased the expression of cleaved caspase 3 in MOLM14 cells.

A

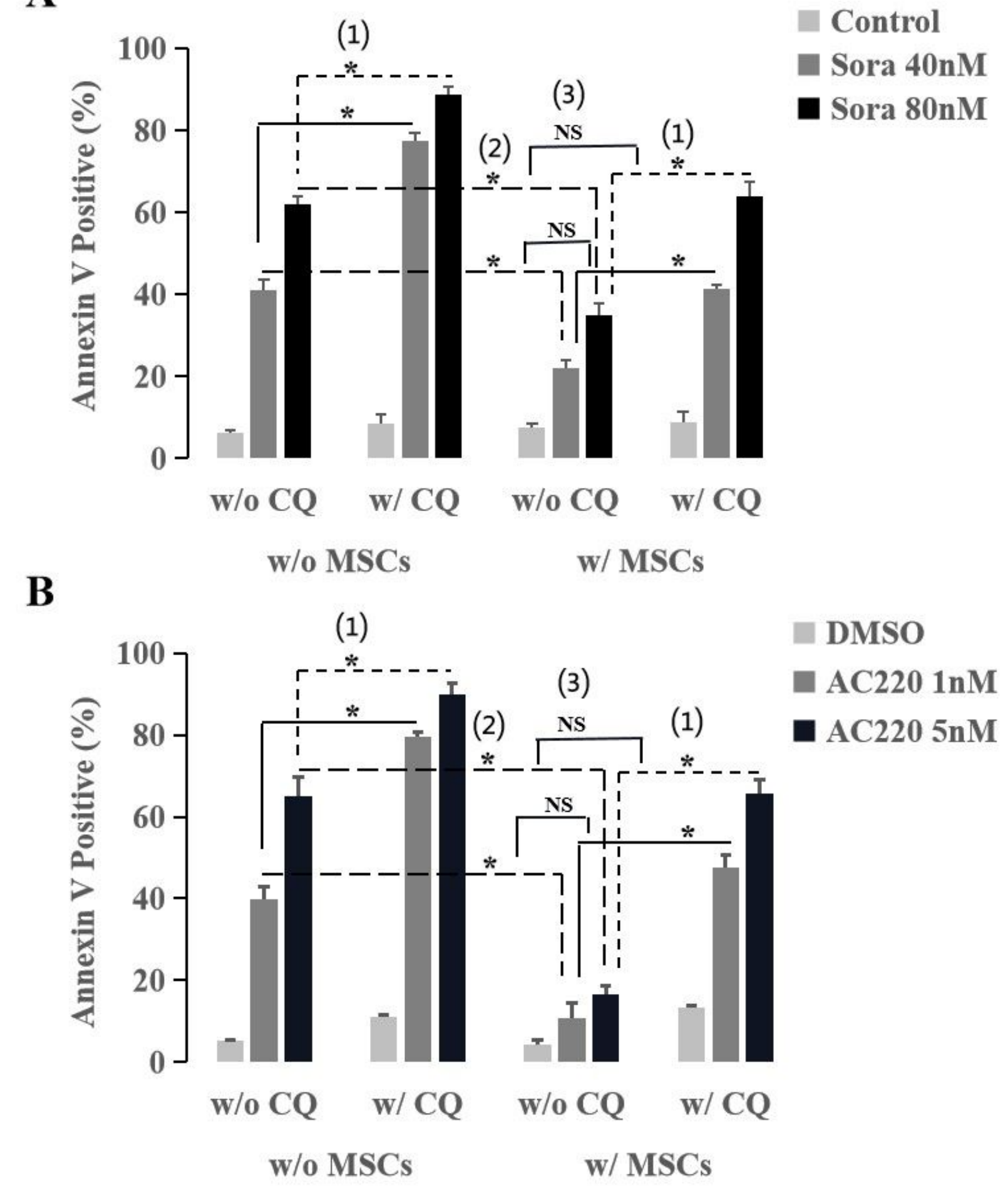

MOLM14

Figure 7

Inhibition of autophagy enhanced the anti-leukemia effect of FLT3 inhibitors and overcame MSCsmediated resistance in FLT3-ITD-positive AML cells. (A1) Inhibition of autophagy enhanced the anti- 
leukemia effect of sorafenib. Regardless of MSCs co-culture, CQ significantly enhanced the killing effect of sorafenib in MOLM14 cells, showing as the apoptosis rate of $41.2 \% \pm 2.5 \% \mathrm{w} / \mathrm{o}$ CQ vs. $77.4 \% \pm 2.1 \% \mathrm{w} /$ CQ $(P=0.004)$ at the concentration of $40 \mathrm{nM}, 62.0 \% \pm 1.9 \%$ vs. $88.7 \% \pm 2.1 \%(P=0.005)$ at $80 \mathrm{nM} \mathrm{w} / \mathrm{o}$ MSCs co-culture; $22.0 \% \pm 2.1 \% \mathrm{w} / \mathrm{o}$ CQ vs. $41.2 \% \pm 1.3 \% \mathrm{w} / \mathrm{CQ}(\mathrm{P}=0.008)$ at $40 \mathrm{nM}, 34.8 \% \pm 3.0 \%$ vs. $63.9 \% \pm 3.7 \%$ $(\mathrm{P}=0.013)$ at $80 \mathrm{nM} \mathrm{w} / \mathrm{MSCs}$ co-culture, respectively. (A2) MSCs decreased the anti-leukemia effect of sorafenib. With MSCs co-culture, the killing effect of sorafenib in MOLM14 cells decreased significantly, showing as the apoptosis rate of $22.0 \% \pm 2.1 \%$ vs. $41.2 \% \pm 2.5 \% \mathrm{w} / \mathrm{o} \mathrm{MSCs}(\mathrm{P}=0.014)$ at $40 \mathrm{nM}$ and $34.8 \%$ $\pm 3.0 \%$ vs. $61.9 \% \pm 1.9 \%(P=0.008)$ at $80 \mathrm{nM}$, respectively. (A3) Inhibition of autophagy overcame MSCsmediated sorafenib resistance. Though co-culture with MSCs, after being dealt with $\mathrm{CQ}$, the killing effect of sorafenib no matter at $40 \mathrm{nM}(41.2 \% \pm 1.3 \%$ vs. $41.2 \% \pm 2.5 \%$ w/o CQ w/o MSCs, $P>0.05)$ or $80 \mathrm{nM}$ $(63.9 \% \pm 3.7 \%$ vs. $62.0 \% \pm 1.9 \% \mathrm{w} / \mathrm{o}$ CQ w/o MSCs, $\mathrm{P}>0.05)$ was similar to that w/o MSCs co-culture. (B1) Inhibition of autophagy enhanced the anti-leukemia effect of AC220. (B2) MSCs decreased the antileukemia effect of AC220. (B3) Inhibition of autophagy overcame MSCs-mediated AC220 resistance. 